\title{
Extracellular vesicles and intercellular communication within the nervous system
}

\author{
Valentina Zappulli, ${ }^{1}$ Kristina Pagh Friis, ${ }^{2}$ Zachary Fitzpatrick, ${ }^{2}$ Casey A. Maguire, ${ }^{2}$ and Xandra O. Breakefield ${ }^{2,3}$ \\ 'Department of Comparative Biomedicine and Food Science, University of Padova, Legnaro, Italy. ${ }^{2}$ Department of Neurology and ${ }^{3}$ Center for Molecular Imaging Research, Department of Radiology, \\ Massachusetts General Hospital and NeuroDiscovery Center, Harvard Medical School, Boston, Massachusetts, USA.
}

\begin{abstract}
Extracellular vesicles (EVs, including exosomes) are implicated in many aspects of nervous system development and function, including regulation of synaptic communication, synaptic strength, and nerve regeneration. They mediate the transfer of packets of information in the form of nonsecreted proteins and DNA/RNA protected within a membrane compartment. EVs are essential for the packaging and transport of many cell-fate proteins during development as well as many neurotoxic misfolded proteins during pathogenesis. This form of communication provides another dimension of cellular crosstalk, with the ability to assemble a "kit" of directional instructions made up of different molecular entities and address it to specific recipient cells. This multidimensional form of communication has special significance in the nervous system. How EVs help to orchestrate the wiring of the brain while allowing for plasticity associated with learning and memory and contribute to regeneration and degeneration are all under investigation. Because they carry specific disease-related RNAs and proteins, practical applications of EVs include potential uses as biomarkers and therapeutics. This Review describes our current understanding of EVs and serves as a springboard for future advances, which may reveal new important mechanisms by which EVs in coordinate brain and body function and dysfunction.
\end{abstract}

\section{Introduction}

Recent research has revealed an expanded range of modes of communication among cells, which includes not only the secretome (organic molecules and inorganic elements), but also vesicular and particulate carriers, which contain proteins, lipids, and nucleic acids that are not soluble or are unstable in the extracellular environment on their own. This new modality is exemplified by extracellular vesicles (EVs), which are produced by virtually all cells, have various means of biogenesis, carry different cargoes, and change dynamically in number and content in response to physiologic and environmental conditions. The classification of these vesicle subtypes is ongoing and includes exosomes (30 to $100 \mathrm{~nm}$ in diameter), which are formed from multivesicular bodies; microvesicles (or ectosomes) (100 nm to $1 \mu \mathrm{m}$ in diameter), which bud from the cell surface; oncosomes (ranging from $1 \mu \mathrm{m}$ to $>2 \mu \mathrm{m}$ in diameter), which are large protrusions released from cancer cells through budding; and apoptotic blebs (ranging from $1 \mu \mathrm{m}$ to $>2 \mu \mathrm{m}$ in diameter), which are generated by dying cells (1-4). Throughout this Review the different types of vesicles will be referred to as EVs.

EVs are released from virtually all cell types in the brain, including neural stem/progenitor cells $(5,6)$, neurons (7-9), astrocytes (9-13), oligodendrocytes (13-15), and microglia $(16,17)$ as well as Schwann cells and neurons in the peripheral nervous system (18-20). A schematic overview of potential EV-mediated interactions in the nervous system is provided in Figure 1.

Authorship note: V. Zappulli, K. Pagh Friis, and Z. Fitzpatrick contributed equally to this work.

Conflict of interest: The authors have declared that no conflict of interest exists. Reference information: J Clin Invest. 2016;126(4):1198-1207. doi:10.1172/JCI81134.
The contents of EVs comprise both molecular entities the cells are trying to divest themselves of as well as information packets intended to alter the phenotypic state of other cells. Each vesicle contains multiple proteins, specialized lipids, and selected nucleic acids, such that their effects on recipient cells are combinatorial. As such, it is difficult to factor out the effects of individual components within the vesicles. Interactions with recipient cells, both near to and far from the cells of origin, can include ligand/receptor signaling at the cell surface, fusion of vesicle and plasma membranes, and uptake via endocytosis. The fate of vesicular contents includes degradation or release into the cytoplasm and then transport into the nucleus or cellular membranes, leading to functional consequences. The exchange of vesicles is active and dynamic in both directions among cells, so as to allow response to and coordination of biologic events.

This Review will focus on new areas of research into the action of EVs in the nervous system, including the implications during neural development, synaptic communication, and nerve regeneration. These functions are all essential for maintaining the health of the nervous system. The second part of this Review focuses on the "dark side" of EVs and how EVs can be used to augment the pathology of various neurological diseases. Finally, we provide an account of research into applying EVs to drug and gene therapeutic strategies, focusing on recent developments in the field.

\section{Role in development}

Production of EVs by neural cells during development. The interconnection as well as the maintenance of neuronal circuits depends on a wide variety of interactions between the different cell types in the brain. EVs are an emerging component of these interactions. They appear to have a substantial impact with respect to neural development and genetic variety based on their ability to 


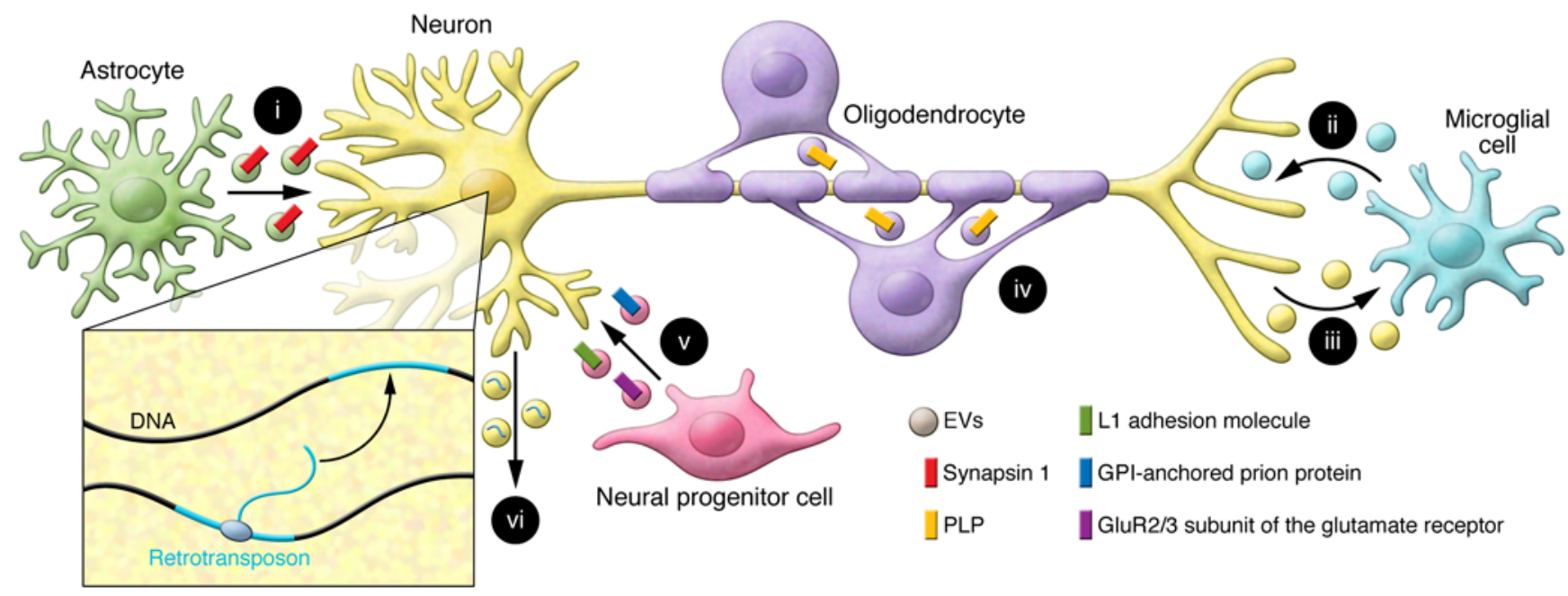

Figure 1. EV-mediated intercellular communication between cells in the nervous system. (i) Astrocyte-derived EVs stimulate dendritic arborization of neurons by transport and release of synapsin I (21); (ii) EVs from microglia increase neuronal synaptic activity, and (iii) neuron-derived EVs activate glial cell functions, such as microglia phagocytosis for clearance of inactive synapses and toxic proteins $(44,148)$. (iv) EVs from oligodendrocytes enhance stress tolerance of neurons, stimulate anterograde transport of signaling molecules in neurons, and carry proteolipoprotein (PLP), which is important for myelination (9, 45). (v) Immature neural progenitor cells release proteins, such as the L1 adhesion molecule, the glycosylphosphatidyl-inositol-anchored (GPI-anchored) prion protein, and the GluR2/3 subunit of the glutamate receptor, via EVs, which participate in early brain development (7). (vi) Retrotransposons can be transported between cells through the EV compartment. During neurogenesis, the activity of retrotransposons is increased, resulting in a high degree of somatic mosaicism in neuronal genomes (31).

transfer various cargoes, such as protein and lipid components, including signaling molecules and transcription factors, as well as DNA and RNA.

As mentioned above, virtually all cell types in the brain have been shown to release EVs; however, the content and effect on neighboring and distant cell types depends on both the donor and recipient cell. In 2005, Marzesco et al. were among the first to report the presence of EVs in the developing brain; they identified EVs in the luminal fluid of the neural tube in embryonic mouse brains (5). EV secretion peaked at around E10.5 to E13.5, and the vesicles could be separated into two sizes, $50-80 \mathrm{~nm}$ and approximately $600 \mathrm{~nm}$, both of which were positive for the stem cell marker prominin-1 (CD133). Subsequently, EVs were reported in neurons cultured from embryos. Following depolarization, immature cortical neurons were found to release proteins, such as the $\mathrm{L} 1$ cell adhesion molecule (L1CAM), the glycosylphosphatidyl-inositol-anchored (GPI-anchored) prion protein, and the glutamate receptor subunit GluR2/3, via EVs, suggesting that EVs regulate synaptic activity (7). More recently, the Bordey laboratory has found evidence supporting a role for EVs in neural development through miRNA-bearing EVs in embryonic cerebrospinal fluid (eCSF) of both rodents and humans (21). The addition of eCSF-derived EVs to neural stem cells activated the mTORC1 pathway, increasing the proliferation rate of these stem cells. These results confirm the presence and functional capacity of EVs in the developing brain, with evolutionary conservation across lower- and higher-order mammalian species.

Another important potential role for EVs in development is the regulation of myelin membrane formation, a complex process that is tightly controlled during both development and regeneration. During development of the CNS, the formation of the myelin membrane is downregulated by EVs released from oligodendrocytes. This downregulation continues until neurons counteract this inhibitory signal- ing with the release of positive signals $(9,13)$. These oligodendrocyte/ neuron interactions show that, in addition to transporting various cargoes, EVs can function in reciprocal collaborations between different cell types. Astrocyte-derived EVs have exhibited a slightly different effect on neighboring cells during brain development, as they have been shown to promote neurite outgrowth and survival of neurons, at least in part through the transfer of synapsin I, thereby supporting the role of glial EVs in neuronal differentiation (22).

The reported effects of EVs on development primarily depend on their cargo. Throughout development, gene expression is regulated by a range of evolutionarily conserved proteins transported through the extracellular environment from donor cells to cells in adjacent tissues. Some of the main cell-fate proteins, such as Hedgehog $(\mathrm{Hh})$, Notch, Wnt, TGF- $\beta$, EGF, and FGF, have been shown to be cargo of EVs (e.g., ref. 23). For example, EV-like particles termed argosomes transport the Wingless $(\mathrm{Wg})$ protein through the disc epithelium during wing development in Drosophila (24). In both human and Drosophila cells, Wnt is found in EVs in association with evenness interrupted/wntless (Evi/WIs), and Wnt signaling is induced in EVrecipient cells $(25,26)$. Budnik and colleagues further demonstrated that the hydrophobic signaling molecules Wnt-1/Wg and their binding partner Evi are released in association with vesicles and that this process is required for Wnt transmission to the postsynaptic muscle cells $(27,28)$. Taken together, these studies in Drosophila support a role for EVs in intercellular communication and as essential regulators of synaptic integrity.

To catch a ride - retrotransposons. Another interesting feature of EVs is their potential to transport mobile DNA/RNA elements. These mobile elements fall into two major classes: DNA transposons, which are inactive in humans, and retrotransposons, which have remained active in both human and murine cells (29). Retrotransposons are remnants of ancient viral infections that have 
insinuated themselves into the genome through infection of germ cells. These elements are scattered throughout the genome and are capable of amplifying themselves as well as changing their position within the genome through RNA intermediates. Among the most significant retrotransposons are the endogenous retrovirus variants, which include long terminal repeat (LTR) retrotransposons and non-LTR retrotransposons, such as long interspersed element 1 (L1) and short interspersed elements (SINEs).

Retrotransposons have been suggested to play a significant role in the development of the nervous system (for review see ref. 30). In the genome of somatic cells, retrotranspositions are more frequent in neural precursors and neurons compared with other neural cell types (31-33). Retrotransposons increase their motility during neurogenesis (31), resulting in a high degree of somatic mosaicism, which may contribute to increased plasticity of neurons in the developing brain. However, insertion of retrotransposons into vulnerable genes can also result in deleterious or tumorpromoting mutations. Many of these mobile DNA sequences have been implicated in various neurological disorders, and increased rates of retrotransposition are seen in murine and human models of Rett syndrome (34) and ataxia telangiectasia (35), which are caused by mutations affecting the epigenetic state of the genome and DNA repair, respectively (36).

Interestingly, retrotransposon elements, including human endogenous retrovirus (HERV) elements, are highly enriched in tumor EVs compared with their cells of origin, which are transferred in vitro to normal human umbilical vein endothelial cells (37). The high activity of the retrotransposons in neural cells during development is thus likely to result in packaging and delivery by EVs between cells; however, this remains to be confirmed in vitro as well as in vivo.

\section{EVs in physiology and pathology of the nervous system}

Synaptic communication. Exosomal EVs are released from endosome-derived multivesicular bodies (MVB) by the soma and the dendrites of mature cortical and hippocampal neurons (8). Their release can be increased in vitro by addition the excitatory neurotransmitter, glutamate (Figure 2A), resulting in enhanced spontaneous electrical activity. AMPA receptor or NMDA receptor antagonists reversed this excitatory synaptic activity, indicating that EV release is modulated by synaptic AMPA and NMDA receptors and, hence, may have a role in normal synaptic physiology. Release of EVs was also enhanced by neuronal depolarization and calcium influx following treatment with the calcium ionophore ionomycin (8), demonstrating the dynamism of this messaging system.

Other in vitro studies report that release of EVs by neurons $(38,39)$ is dependent on synaptic activity and acts as a potential control mechanism for synaptic plasticity and as a component of the neuron-to-neuron communication system $(40,41)$. EVs have also been implicated in controlling retrograde postsynaptic signaling that mediates activity-dependent presynaptic growth and quantal neurotransmitter release at the neuromuscular junction in Drosophila larvae (42). Synaptotagmin 4 (SYT4), which is critical for this retrograde postsynaptic signaling to the motor neuron, is delivered to the postsynaptic terminal in the muscle in EVs released by the presynaptic nerve terminal. This EV communication allows a balancing of synaptic input as the muscle grows.

A potential role of EVs in synaptic function and plasticity is also evidenced by the enrichment within neuronal EVs of proteins, such as synaptic plasticity-associated microtubule-associated protein 1B (MAP1B), and a specific set of activity-related miRNAs (43). The release of neuron-derived EVs from synaptic terminals after depolarization suggests a means of regulating synaptic strength by allowing rapid changes in translation of mRNAs relevant to synaptic activity in the postsynaptic region (ref. 43 and Figure 2A). EVs may also have an important role in regulating synaptic pruning. EVs released by synaptically active neurites appear to participate in eliminating inappropriate synaptic connections (44). Thus, active synapses facilitate the loss of inactive synapses through the release of EVs, which also stimulate production of complement factors and phagocytosis of cellular debris by microglia (44).

Nerve regeneration. Mature oligodendrocytes, which are responsible for myelination of neurons in the brain, release EVs in response to glutamate acting on NMDA and AMPA receptors (9, 45). Oligodendrocyte-derived EVs carry not only specific myelin proteins, such as major myelin proteolipid protein (14), but also other proteins and RNA related to myelination. Internalization of oligodendrocyte-derived EVs by neurons results in an enhanced tolerance to stress, resulting in increased viability $(9,45)$.

In the peripheral nervous system, dedifferentiation and proliferation of Schwann cells, which myelinate peripheral nerve fibers, are key regulators of axon growth and regeneration (46). In this context, Lopez-Verrilli and colleagues demonstrated that Schwann cell-derived EVs stimulated axonal growth and regeneration after nerve damage in vitro when taken up by adjacent sensory neurons (ref. 19 and Figure 2B). After internalization in damaged axons, Schwann cell-derived EVs modulated growth cone morphology and downregulated growth inhibitors, such as RhoA GTPase, both in culture and in vivo. In addition, these EVs transferred newly synthesized ribosomal RNA and mRNAs to axons, which increased axonal protein synthesis at the damaged site (refs. 18, 47, and Figure 2B). Schwann cell-derived miRNAs were also observed in axon terminals, supporting the possibility of direct transfer via EVs, with subsequent effects on coordination of mRNA translation and neurite growth (48-50).

Neurodegeneration. While they play crucial roles in physiological processes, EVs/exosomes also contribute to the development of disease states. Neurodegenerative diseases, including Alzheimer's disease (AD), Parkinson's disease (PD), and prion diseases, are characterized by protein aggregation and deposition in specific brain regions. However, EVs appear to act at cross-purposes in neurodegeneration, particularly in their role in amyloid formation and clearance. On the one hand, EVs appears to aid in the formation and the spread of the toxic amyloid proteins across the different regions of the brain, while on the other hand they can also serve as a means for disassembly and clearance of toxic proteins by phagocytic cells (Figure 2C). EVs have been implicated in a number of neurodegenerative diseases, including prion-induced spongiform encephalopathy and amyloid- $\beta$ peptide-related (A $\beta$-related) AD. As a corollary, these toxic protein-containing EVs may prove useful as biomarkers for these disease states. 
A

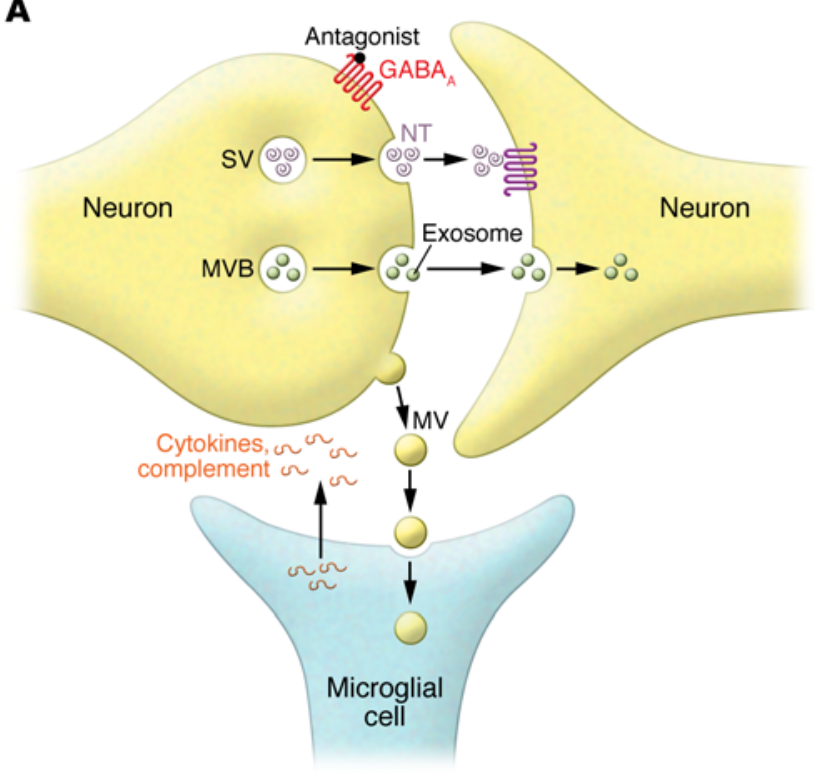

B

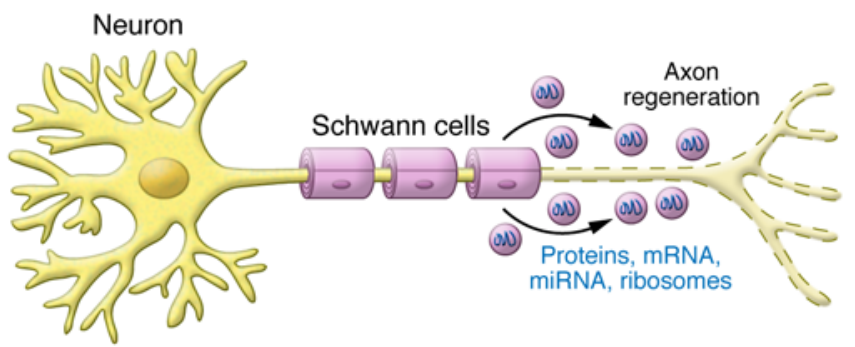

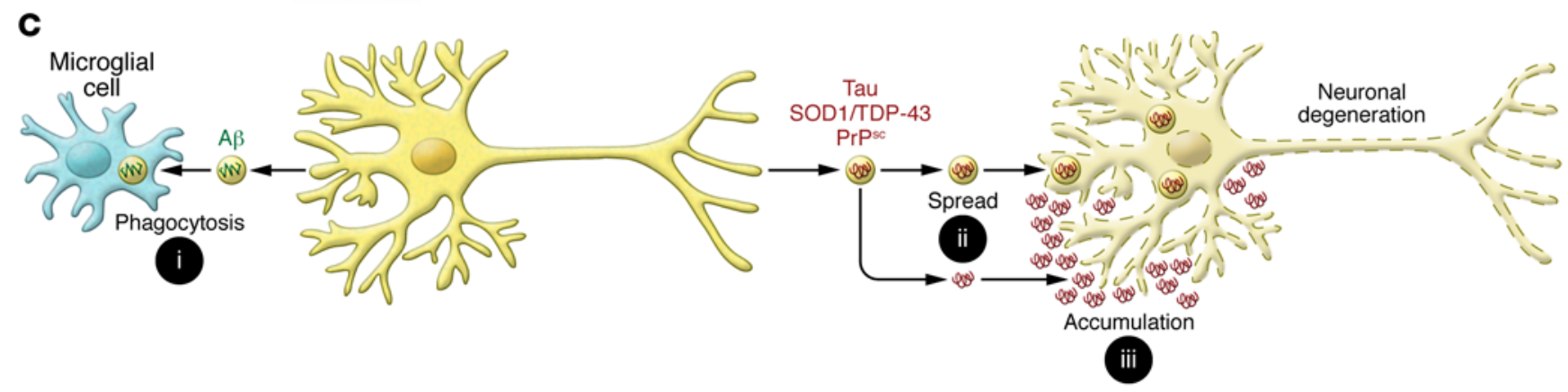

Figure 2. EVs in nervous system physiology and pathology. (A) The role of EVs in synaptic communication. The addition of GABA $\mathrm{A}_{A}$ receptor antagonists results in the release of presynaptic EVs that are taken up by postsynaptic cells and modulate synaptic strength and retrograde signaling. They can also activate glial functions, such as microglia phagocytosis for clearance of inactive synapses and release of cytokines and complement. Synaptic vesicles (SV) fuse with the plasma membrane to release neurotransmitters (NT) into the extracellular space, which bind to receptors on postsynaptic neurons, while EVs are released intact into this space by fusion of multivesicular bodies (MVB) with the plasma membrane or budding from the plasma membrane. (B) The role of EVs in axonal regeneration in the peripheral nervous system is mediated by Schwann cells that release EVs containing proteins, miRNA, mRNA, and ribosomes to promote axonal growth. (C) In the pathogenesis of neurodegenerative diseases, (i) EVs can modulate phagocytic clearing of misfolded proteins, such as that described for A $\beta$ in AD. On the other hand, EVs can promote extracellular release, (ii) cell-to-cell spreading, and (iii) accumulation of toxic proteins such as tau, SOD1, TDP-43, and prions, all of which are associated with neuronal degeneration.

Accumulation and cell-to-cell transmission of infectious misfolded proteins, such as the scrapie form of prion protein $(\operatorname{PrPsc})$, are known to be key mechanisms in some prion diseases. Both normal $\left(\mathrm{PrP}^{\mathrm{c}}\right)$ and pathological $\left(\operatorname{PrP}^{\mathrm{sc}}\right)$ prion proteins are incorporated into EVs by normal and prion-infected cells $(51,52)$. PrPsc-carrying EVs are present in biological fluids (i.e., blood) of infected animals $(53,54)$, and these PrPsc-carrying EVs can spread prion infection to normal recipient cells (55). These findings have opened up new and important insights into the complicated mechanisms of transmission and propagation of prion diseases.

The $\mathrm{AD} A \beta$ peptide is produced in the early endosomal compartment and is released from the cells via exosomes. Immunohistochemical analysis in brain sections from patients with $\mathrm{AD}$ and patients with PD and age-matched control subjects showed enrichment of the exosomal marker Alix around small neuritic plaques and in large diffuse plaques in brain sections from all patients with $\mathrm{AD}$ tested (56). These results suggest that exosomes may act as nucleation centers for amyloid plaque formation, and two recent studies $(57,58)$ demonstrated that exosomal/intraluminal vesicles accelerate amyloid formation. At present there is some controversy in the field as to the role of EVs in AD; while some studies (59-61) suggest that exosome-associated $\mathrm{A} \beta$ is protective, other studies suggest that exosome-associated $A \beta$ contributes neurotoxic amyloid formation (56, 62-65). Moreover, misfolded tau protein, which is implicated in $\mathrm{A} \beta$ aggregate formation, may also be released from neurons via EVs (66). EV release of misfolded tau appears to recruit other proteins into EVs, including mitochondrial-, synaptic-, and axonogenesis-related proteins that have been linked to the pathogenesis of $\mathrm{AD}$ and other neurodegenerative diseases (i.e., tauopathies) (66). In PD, the accumulation of misfolded $\alpha$-synuclein has been associated with EV release by neurons $(67,68)$; however, in $\mathrm{PD}$ as well as other neurodegenerative processes, the potential pathways of cell-to-cell spreading and disease propagation are still being evaluated $(68,69)$.

Amyotrophic lateral sclerosis (ALS) is caused by progressive accumulation of a misfolded mutant form of superoxide dis- 
mutase 1 (SOD1) in a subset of patients and by cytosolic translocation and misfolding of TAR DNA-binding protein-43 (TDP-43) in others (70). A growing body of evidence suggests that, particularly for SOD1 but also for TDP-43, EVs are the potential mechanism of extracellular spread and cell-to-cell propagation within the brain, serving to extend the range of toxicity and cell damage $(70,71)$. TDP-43 is especially interesting in this regard, as it is an RNAbinding protein associated with ribonuclear protein particles (72), which have been implicated in the process of incorporating RNA into EVs (73) and in suppressing HERV expression, which is elevated in patients with ALS with TDP-43 mutations (74).

Although a subgroup of neurodegenerative diseases results from expression of mutant proteins, many others may be a result of biologic processes induced by injury to the brain (75). An important aspect of the inflammatory response to injury is the association between microparticles (EVs) and IL-1 $\beta$ cytokine release from microglia and astrocytes (11). In parallel, it appears that hematopoietic cells can influence the genome of neurons, as demonstrated by the ability of Cre recombinase (mRNA and protein) in EVs produced by hematopoietic cells to activate floxed reporters in the genome of neurons in vitro and in the brain as a result of inflammation, suggesting a very insidious means of intrusion into neuronal integrity (76). EVs released by microglia and endothelial cells in the brain have also been implicated in breaking down the blood-brain barrier and promoting influx of immune cells in multiple sclerosis (MS; ref. 77).

A new effort is underway to use RNA and protein cargo in biofluid-derived EVs as a means to monitor neurologic disease status. The number and content of EVs isolated from cerebrospinal fluid (CSF) could aid in monitoring AD and PD status (78). CSF EVs from patients with AD have abnormally high levels of phosphorylated tau (79), while those from patients with MS have high levels of isolectin B4 (IB4), which is associated with neuroinflammation (80). CSF EVs from patients with ALS have elevated TDP-43 (81). Increased levels of the PD-associated proteins deglycase DJ-1 and leucine-rich repeat kinase 2 (LRRK2) have been found in bloodderived EVs in patients with PD $(82,83)$. Brain-derived EVs in the blood represent a small fraction of the total EV content, most of which is derived from vascular-associated cells (84). Using immunoaffinity capture with L1CAM antibodies to isolate EVs from the blood, Shi et al. (85) found elevated levels of $\alpha$-synuclein in EVs from patients with PD compared with controls, and Fiandaca et al. (86) found elevated tau, phosphorylated tau, and $A \beta 42$ in patients with AD compared with controls. More work is needed to sort out the most effective means of enriching for brain-derived EVs from biofluids and to determine the most informative target proteins or RNAs with respect to disease status for different neurodegenerative diseases (87).

\section{Brain tumors}

Glioblastoma takes over the brain with EVs as armaments. Glioblastoma $(\mathrm{GBM})$ represents the most malignant brain tumor and one of the most untreatable cancers (88). For GBMs, some of the "secrets of their success" lie in their genetic and phenotypic heterogeneity, with a stash of cancer stem cells that are resistant to treatment, as well as their ability to subvert the normal tissue environment to support their growth and expansion. Although their secretome and cell-to-cell contacts are clearly critical components, recent studies implicate EV-mediated intercellular communication in GBM. Numerous studies have demonstrated that GBM-derived EVs promote tumor cell proliferation, angiogenesis, invasion, and suppression of antitumor immune responses (refs. 1, 2, and Figure 3). Many aspects of this vesicular messaging service remain to be fully elucidated, i.e., the different subtypes of vesicles released from various cells in the tumor environment, the contents of these vesicles, and the function of the vesicle contents. Tumor cells exhibit enhanced vesicle release compared with most normal cells (89) and release of unique types of vesicles, such as HERV particles (37) and oncosomes (90). The enrichment of retroviral retrotransposon sequences in tumor vesicles indicates the potential for cell-to-cell transfer of mobile elements through EVs, which could result in genetic variation and increased cellular plasticity in the tumor microenvironment. EVs also serve to dispose of molecules that inhibit tumor growth, e.g., miR-1, which targets annexin A2 (91), a mediator of tumor cell invasion, and miR-451, which targets calcium-binding protein 39 (CAB39), a negative regulator of the oncogenic PI3K/AKT pathway (92). EVs also promote drug resistance through transfer of P-glycoprotein (93). Although many $\mathrm{EV}$ messages may be lost or degraded in recipient cells, functional transfer of oncogenic proteins, such as EGFRvIII (94) and transglutaminase (95), has been confirmed. Additionally, mRNA and miRNAs transferred by EVs can be translated (e.g., ref. 96) and inhibit mRNA translation (97), respectively. There is mounting evidence that EVs carry informative molecules that can change the transcriptome and possibly the epigenetic state of recipient cells. For example, deep sequencing of RNA from GBM EVs reveals a host of small noncoding regulatory RNAs; treatment of brain microvascular endothelial cells with these EVs has marked effects on their transcriptome (98) and is associated with increased tubule formation $(98,99)$. For example, there is rapid recovery of tumor growth following radiation and chemotherapy, which kill most of the more differentiated tumor cells. This recovery is thought to be mediated by glioma stem cells (GSCs) (89). GSCs release EVs (100) that contain regulatory RNAs and transcription factors that may be able to reset the epigenetic status of surviving "differentiated" tumor cells, rejuvenating them and stimulating tumorigenesis (101) as well as reprogramming normal cells in the environment to increase their plasticity and responsiveness to tumor signals. The tumor transition is typified by a shift from a less aggressive, proneural subtype of GBM to a more aggressive mesenchymal subtype (102), which may be mediated in part by GSC EVs (89). Changes in the tumor microenvironment are undoubtedly achieved through combinatorial "armaments," as recipient cells are bombarded not only with EVs containing many proteins, lipids, and RNA species, but also with the secretome of the tumor and potentially with tunneling nanotubes opening direct communication between cells (103).

Communication between GBM EVs and the body. Although GBM cells usually remain within the brain proper and do not metastasize, they do influence cells outside the brain. Circulating GBM tumor cells were detected in blood of at least one patient with the mesenchymal subtype of GBM (104); thus, the failure to metastasize may reflect the rapid lethality of these brain tumors rather than the lack of peripheral dissemination 


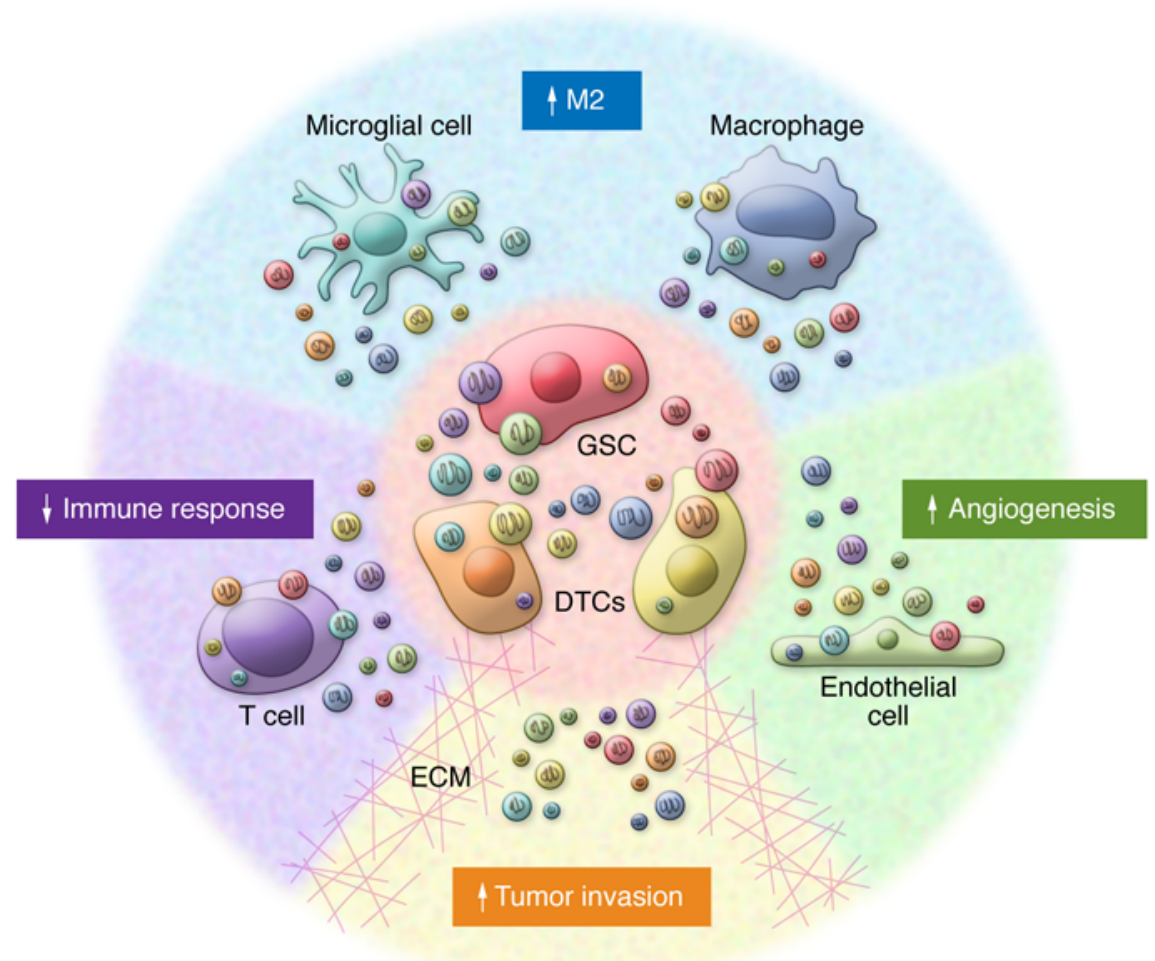

Figure 3. Vesicular exchange in the brain tumor microenvironment. GBM tumors are made up of a heterogeneous group of genetically related cancer cells, shown here as two types of differentiated tumor cells (DTCs) within the same tumor. Tumors also contain glioma stem cells (CSCs), which tend to be resistant to therapy and are thought to rejuvenate differentiated tumor cells after therapy. Tumor cells release at least three types of vesicles - exosomes, microvesicles, and large oncosomes. Normal cells in the brain tumor environment include T cells, microglia, macrophages, and endothelial cells, which also release exosomes and microvesicles. The secretome and EVs of the tumors modulate the phenotype of these normal cells, including promoting an immune-repressive T cell Th2 phenotype, stimulating microglia and macrophages to assume the M2-activated state in support of tumor progression, and inducing endothelial cell-mediated angiogenesis as well as opening up the extracellular matrix (ECM) to facilitate invasion of cancer cells. Vesicles, shown with color coding that matches the cell of origin, are exchanged among all cells in the environment. They all contain a cell-specific cargo of lipids, proteins, and nucleic acids, which are delivered into recipient cells.

of tumor cells. Gliomas also secrete chemoattractants, which stimulate monocytes to enter the brain where they are converted to macrophages that, together with M2-activated microglia, set up a protective zone around the tumor (105). Once within the brain these myeloid cells actively take up GBM EVs, resulting in changes in their phenotype that are correlated, at least in part, with transfer of miRNAs, such as miR-451 and miR-21 (97). EVs and cytokines from serum from a patient with GBM can act in concert to convert circulating monocytes to a M2-like activation state and promote a Th2 bias, thereby decreasing the ability of immune cells to destroy tumor cells (refs. 106, 107, and Figure 3). GBMs are also associated with a peripheral coagulopathy (108), which is mediated by microparticles (EVs) released from the tumors that contain high levels of tissue factor and initiate thrombin formation $(109,110)$.

GBM EVs as biomarkers - telltale signs. The finding of EVs in the sera of patients with GBM carrying the mutant EGFRvIII mRNA in tumors opened the door for using EV contents in biofluids as biomarkers for brain tumors and other cancers (99). The list of potential biomarkers that can provide information on glioma status and response to therapy is expanding and includes changes in the transcriptome profile of $\operatorname{EV} \operatorname{mRNA}(111,112)$ and small noncoding RNAs in sera (113) as well as mutations in isocitrate dehydrogenase (IDH) mRNA (114) and elevated miR-21 levels in CSF EVs (ref. 115 and for review see ref. 88). Deepsequencing methods will increase the sensitivity of detection of RNA biomarkers. High-resolution, point-of-care technologies have also been developed to monitor levels of GBM-associated proteins (116) and mRNAs in serum EVs that are predictive of drug response (117).

\section{Putting EVs to work - naturals at therapeutic delivery}

Therapeutic application of unmodified EVs. The diverse roles of EVs in the normal and pathological states of nervous system development and physiology highlight their potential as therapeutic modalities for neurological disease and injury. EVs are safer than the therapeutic cells from which they are derived, as they cannot replicate or directly form a tumor. However, a single dose of EVs may have only transient therapeutic effects. Mesenchymal stem cell-based (MSC-based) therapies remain at the forefront of regenerative medicine, as they have demonstrated clinical and preclinical efficacy (118-121). Accumulating evidence suggests that the regenerative effects of MSCs are partially attributable to their EVs (122), which may retain the immunological properties that make them appropriate for allogeneic use (123). EVs from MSCs cocultured with ischemic brain tissue from rats subjected to middle cerebral artery occlusion promoted neurite remodeling in culture (124). Systemic delivery of MSC-derived EVs induced functional recovery and neurovascular plasticity in a rat stroke model, with neurite outgrowth effects partly mediated by EV-associated miRNA-133b (125). Undoubtedly other EV cargo also contributes to this MSC EV-mediated neural regeneration. The multifaceted nature of EV cargo underscores an advantage of utilizing EVs for therapy - they carry a repertoire of bioactive molecules with a combinatorial capacity that would be challenging to recapitulate by artificial means.

Other reports have explored the use of unmodified EVs as therapeutics in neurodegenerative conditions, such as $\mathrm{AD}$ and MS. As discussed, A $\beta$ peptide is released in association with exo- 
somes, supporting a role for EVs in A $\beta$ formation. This observation led Yuyama et al. to explore the effects of infusion of neuroblastoma-derived EVs into the cerebra of transgenic mice overexpressing amyloid peptide precursor (60). This infusion resulted in EV-mediated scavenging and subsequent degradation of $\mathrm{A} \beta$ by microglia as well as reduced $A \beta$-mediated synaptotoxicity.

As a potent source of EVs, DCs release vesicles with characteristics dependent on external stimuli and cell state (126). EVs from primary rat bone marrow-derived DCs stimulated with IFN- $\gamma$ (IFN- $\gamma$-DC-EVs) were preferentially taken up by oligodendrocytes and enhanced myelination and oxidative tolerance in hippocampal slice cultures and improved recovery from MS-like demyelination following acute lysolecithin-induced demyelination (127). Intranasal delivery of IFN- $\gamma$-DC-EVs in rats resulted in increased myelination in the brain in vivo. miRNA-219, which has been implicated in oligodendrocyte differentiation and antiinflammatory pathways, was enriched in IFN- $\gamma$-DC-EVs, suggesting that it plays a role in EV-mediated remyelination.

Potential of loading EVs with therapeutic agents for neurologic disease applications. Much research is devoted to loading EVs with biological cargo (128). EVs represent an attractive platform for the delivery of functional cargoes to the CNS, due to their natural ability to shuttle biomolecules intercellularly, to traverse biological barriers, and to protect intraluminal contents. Strategies for loading are being optimized and include two main approaches: EV packaging by donor cells, with passive incorporation of typically high concentrations of therapeutic agents present in the cells, and loading of isolated EVs using different bioengineering techniques. Recently, different molecules have been loaded onto/into EVs as therapeutics, including drugs (129), siRNA (130), miRNA (131), mRNA/protein (132), plasmid DNA (133), proteins (134), natural compounds (135), and viral particles (136).

Although limited in number, there has been an increase in studies with EV-based therapeutics in the CNS. Zhuang et al. delivered EVs loaded with antiinflammatory curcumin intranasally into mice to suppress LPS-induced brain inflammation (137). The proposed mechanism for immune suppression was microglial uptake of curcumin-loaded EVs. In another approach, Munoz et al. showed that MSCs transfected with anti-miR-9 could transfer this siRNA via EVs to glioma cells in culture and block miR-9-mediated chemotherapy resistance (138). Yang et al. (139) successfully treated a zebrafish model of primary brain cancer by systemically administering EVs from a murine brain endothelial cell line premixed with doxorubicin and paclitaxel (139). Another group reported that murine DC-derived EVs, which were membranetagged with the neurotropic rabies virus glycoprotein peptide and electroporated with siRNAs, could decrease mRNA levels in the brain after systemic injection (130). It is clear that EVs can be targeted to the brain and can carry a wide variety of therapeutics, including their own natural cargo.

Vexosomes - the AAV-EV combination. Adeno-associated virus (AAV) vectors are the lead gene therapy candidates for neurological diseases. Clinical trials using AAV vectors are being evaluated for many CNS disorders, with clinical benefit reported in some trials (140). Despite this progress, barriers exist for gene therapy vectors (for review see ref. 141). One bar- rier encountered by AAV vectors is the presence of preexisting antibodies from prior exposure to the natural virus (142), and even low titers of antibodies can prevent efficient transduction when vector is delivered peripherally (143).

Viruses also exploit EVs to hide from patient-derived antibodies $(144,145)$, and some AAV vectors associate with EVs during production in 293T cells (136). Exosome-associated AAVs, termed vexosomes, efficiently transfer genes in cultured cells. In contrast to standard AAV, systemically injected vexosomes can evade antiAAV-neutralizing antibodies in mice (128). Another limitation of $\mathrm{AAV}$ vectors is targeting to specific tissues. By tagging the surface of vexosomes with a neurotropic peptide it is possible to enhance brain transduction following peripheral delivery (128). Because the AAV component of vexosomes allows long-term transgene expression in the CNS, it may be preferable to use vexosomes rather than EVs for delivery in certain applications (146).

\section{Conclusion}

Increasing evidence supports an important role for EVs in intercellular communication in the nervous system. EVs regulate various physiological and pathological processes, including development, synaptic neurotransmission, nerve regeneration, neurodegeneration, and brain tumor progression (147). EV contents can serve as modulators of the physiology of donor and recipient cells, both by what is released and what is taken up, with exchange occurring in both directions. Mechanistically EVs participate in clearance of substances, information exchange, and epigenetic modulation and may even be responsible for spreading of pathological proteins in some neurodegenerative diseases. This complex, multifunctional activity is explained by the highly heterogeneous content of EVs, including cytoplasmic and membranous proteins, noncoding regulatory RNAs, and genomic and mitochondrial DNA as well as the diverse types of cells that are capable of both releasing and entrapping EVs in the nervous system (2). Changes induced by these vesicles can be transitory or long term and can serve as instructions that determine genomic and phenotypic status. EVs are increasingly being used as biomarkers of disease, as even those generated in the nervous system end up in biofluids, and their inherent capacity for delivery throughout the body is being harnessed for therapeutic purposes.

\section{Acknowledgments}

We thank Suzanne McDavitt for skilled editorial assistance, Lawrence Rajendran for insights into the role of EVs in neurodegeneration, and Sybren Maas for insights into the role of EVs in brain tumors. This work was supported by the NIH Common Fund through the Office of Strategic Coordination/Office of the NIH Director (NCI U19 CA179563 and NIH/NCI P01 CA069246 to X.O. Breakefield); the Alfred Benzon Foundation (to K.P. Friis); the Fulbright Visiting Scholar Program Institute of International Education (to V. Zappulli); and an American Brain Tumor Association Discovery Grant (to C.A. Maguire).

Address correspondence to: Xandra Breakefield, Massachusetts General Hospital, CNY Building 149, Room 6203, 149 13th Street, Charlestown, Massachusetts, 02129, USA; Phone: 617.726.5728; E-mail: xandra_breakefield@hms.harvard.edu. 
1. D'Asti E, Garnier D, Lee TH, Montermini L, Meehan B, Rak J. Oncogenic extracellular vesicles in brain tumor progression. Front Physiol. 2012;3:294.

2. van der Vos KE, Balaj L, Skog J, Breakefield XO. Brain tumor microvesicles: Insights into intercellular communication in the nervous system. Cell Mol Neurobiol.2011;31(6):949-959.

3. Cocucci E, Meldolesi J. Ectosomes and exosomes: shedding the confusion between extracellular vesicles. Trends Cell Biol. 2015;25(6):364-372.

4. Rilla K, Siiskonen H, Tammi M, Tammi R. Hyaluronan-coated extracellular vesicles - a novel link between hyaluronan and cancer. Adv Cancer Res. 2014;123:121-148.

5. Marzesco AM, et al. Release of extracellular membrane particles carrying the stem cell marker prominin-1 (CD133) from neural progenitors and other epithelial cells. J Cell Sci. 2005;118(pt 13):2849-2858.

6. Yuan A, et al. Transfer of microRNAs by embryonic stem cell microvesicles. PLoS One. 2009;4(3):e4722.

7. Fauré J, et al. Exosomes are released by cultured cortical neurones. Mol Cell Neurosci. 2006;31(4):642-648.

8. Lachenal G, et al. Release of exosomes from differentiated neurons and its regulation by synaptic glutamatergic activity. Mol Cell Neurosci. 2011;46(2):409-418.

9. Frühbeis C, et al. Neurotransmitter-triggered transfer of exosomes mediates oligodendrocyte-neuron communication. PLoS Biol. 2013;11(7):e1001604.

10. Taylor AR, Robinson MB, Gifondorwa DJ, Tytell M, Milligan CE. Regulation of heat shock protein 70 release in astrocytes: role of signaling kinases. Dev Neurobiol. 2007;67(13):1815-1829.

11. Bianco F, et al. Acid sphingomyelinase activity triggers microparticle release from glial cells. EMBO J. 2009;28(8):1043-1054.

12. Guescini M, Genedani S, Stocchi V, Agnati LF. Astrocytes and glioblastoma cells release exosomes carrying mtDNA. J Neural Transm. 2010; 117(1): 1-4.

13. Bakhti M, Winter C, Simons M. Inhibition of myelin membrane sheath formation by oligodendrocytederived exosome-like vesicles. J Biol Chem. 2011;286(1):787-796.

14. Krämer-Albers EM, et al. Oligodendrocytes secrete exosomes containing major myelin and stress-protective proteins: Trophic support for axons? Proteomics Clin. 2007;1(11):1446-1461.

15. Fitzner D, et al. Selective transfer of exosomes from oligodendrocytes to microglia by macropinocytosis. JCell Sci. 2011;124(pt 3):447-458.

16. Potolicchio I, et al. Proteomic analysis of microgliaderived exosomes: metabolic role of the aminopeptidase CD13 in neuropeptide catabolism. J Immunol. 2005;175(4):2237-2243.

17. Bianco F, et al. Astrocyte-derived ATP induces vesicle shedding and IL-1 beta release from microglia. JImmunol. 2005;174(11):7268-7277.

18. Lopez-Verrilli MA, Court FA. Transfer of vesicles from schwann cells to axons: a novel mechanism of communication in the peripheral nervous system. Front Physiol. 2012;3:205.

19. Lopez-Verrilli MA, Picou F, Court FA. Schwann cell-derived exosomes enhance axonal regeneration in the peripheral nervous system. Glia. 2013;61(11):1795-1806.

20. Fevrier B, et al. Cells release prions in association with exosomes. Proc Natl Acad Sci U S A. 2004;101(26):9683-9688.

21. Feliciano DM, Zhang S, Nasrallah CM, Lisgo SN, Bordey A. Embryonic cerebrospinal fluid nanovesicles carry evolutionarily conserved molecules and promote neural stem cell amplification. PLoS One. 2014;9(2):e88810.

22. Wang $S$, et al. Synapsin I is an oligomannose-carrying glycoprotein, acts as an oligomannose-binding lectin, and promotes neurite outgrowth and neuronal survival when released via glia-derived exosomes. J Neurosci. 2011;31(20):7275-7290.

23. Kalra H, et al. Vesiclepedia: a compendium for extracellular vesicles with continuous community annotation. PLoS Biol. 2012;10(12):e1001450.

24. Greco V, Hannus M, Eaton S. Argosomes: a potential vehicle for the spread of morphogens through epithelia. Cell. 2001;106(5):633-645.

25. Gross JC, Chaudhary V, Bartscherer K, Boutros M. Active Wnt proteins are secreted on exosomes. Nat Cell Biol. 2012;14(10):1036-1045.

26. Gross JC, Boutros M. Secretion and extracellular space travel of Wnt proteins. Curr Opin Genet Dev. 2013;23(4):385-390.

27. Koles K, et al. Mechanism of evenness interrupted (Evi)-exosome release at synaptic boutons. J Biol Chem. 2012;287(20):16820-16834.

28. Korkut C, et al. Trans-synaptic transmission of vesicular Wnt signals through Evi/Wntless. Cell. 2009;139(2):393-404

29. Levin HL, Moran JV. Dynamic interactions between transposable elements and their hosts. Nat Rev Genet. 2011;12(9):615-627.

30. Erwin JA, Marchetto MC, Gage FH. Mobile DNA elements in the generation of diversity and complexity in the brain. Nat Rev Neurosci. 2014;15(8):497-506.

31. Muotri AR, et al. Somatic mosaicism in neuronal precursor cells mediated by $\mathrm{L} 1$ retrotransposition. Nature. 2005;435(7044):903-910.

32. Coufal NG, et al. L1 retrotransposition in human neural progenitor cells. Nature. 2009;460(7259):1127-1131.

33. Bundo $\mathrm{M}$, et al. Increased 11 retrotransposition in the neuronal genome in schizophrenia. Neuron. 2014;81(2):306-313

34. Muotri AR, et al. L1 retrotransposition in neurons is modulated by MeCP2. Nature. 2010;486(7322):443-446.

35. Coufal NG, et al. Ataxia telangiectasia mutated (ATM) modulates long interspersed element-1 (L1) retrotransposition in human neural stem cells. Proc Natl Acad Sci U S A. 2011;108(51):20382-20387.

36. Reilly MT, Faulkner GJ, Dubnau J, Ponomarev I, Gage FH. The role of transposable elements in health and diseases of the central nervous system. JNeurosci. 2013;33(45):17577-17586.

37. Balaj L, et al. Tumour microvesicles contain retrotransposon elements and amplified oncogene sequences. Nat Commun. 2011;2:180.

38. Ghidoni R, et al. Cystatin $\mathrm{C}$ is released in association with exosomes: a new tool of neuronal communication which is unbalanced in Alzheimer's disease. Neurobiol Aging. 2011;32(8):1435-1442.

39. Putz U, et al. Nedd 4 family-interacting protein 1 (Ndfip1) is required for the exosomal secretion of Nedd 4 family proteins. J Biol Chem. 2008;283(47):32621-32627.

40. Chivet M, et al. Exosomes as a novel way of interneuronal communication. Biochem Soc Trans. 2013;41(1):241-244

41. Edelstein L, Smythies J. The role of epigeneticrelated codes in neurocomputation: dynamic hardware in the brain. Philos Trans R Soc Lond B Biol Sci. 2014;369(1652): 20130519.

42. Korkut C, et al. Regulation of postsynaptic retrograde signaling by presynaptic exosome release. Neuron. 2013;77(6):1039-1046.

43. Goldie BJ, et al. Activity-associated miRNA are packaged in Map1b-enriched exosomes released from depolarized neurons. Nucleic Acids Res. 2014;42(14):9195-9208.

44. Bahrini I, Song JH, Diez D, Hanayama R. Neuronal exosomes facilitate synaptic pruning by up-regulating complement factors in microglia. Sci Rep. 2015;5:7989.

45. Fröhlich D, et al. Multifaceted effects of oligodendroglial exosomes on neurons: impact on neuronal firing rate, signal transduction and gene regulation. Philos Trans R Soc Lond B Biol Sci. 2014;369(1652).

46. Bosse F. Extrinsic cellular and molecular mediators of peripheral axonal regeneration. Cell Tissue Res. 2012;349(1):5-14.

47. Sotelo JR, et al. Glia to axon RNA transfer. Dev Neurobiol. 2014;74(3):292-302.

48. Chang LW, et al. An integrated approach to characterize transcription factor and microRNA regulatory networks involved in Schwann cell response to peripheral nerve injury. BMC Genomics. 2013;14:84.

49. Svaren J. MicroRNA and transcriptional crosstalk in myelinating glia. Neurochem Int. 2014;77:50-57.

50. Zhang Y, et al. The MicroRNA-17-92 cluster enhances axonal outgrowth in embryonic cortical neurons. J Neurosci. 2013;33(16):6885-6894.

51. Castro-Seoane R et al. Plasmacytoid dendritic cells sequester high prion titres at early stages of prion infection. PLoS Pathog. 2012;8(2):e1002538.

52. Klöhn PC, Castro-Seoane R, Collinge J. Exosome release from infected dendritic cells: a clue for a fast spread of prions in the periphery? J Infect 2013;67(5):359-368.

53. Vella LJ, Greenwood DL, Cappai R, Scheerlinck JP, Hill AF. Enrichment of prion protein in exosomes derived from ovine cerebral spinal fluid. Vet Immunol Immunopathol. 2008;124(3-4):385-393.

54. Properzi F, et al. Detection of exosomal prions in blood by immunochemistry techniques. J Gen Virol. 2015;96(pt 7):1969-1974.

55. Lever E, Sheer D. The role of nuclear organization in cancer. J Pathol. 2010;220(2):114-125.

56. Rajendran L, et al. Alzheimer's disease $\beta$-amyloid peptides are released in association with exosomes. Proc Natl Acad Sci U S A 2006;103(30):11172-11177.

57. van Niel G, et al. Apolipoprotein E regulates amyloid formation within endosomes of pigment cells. Cell Rep. 2015;13(1):43-51.

58. Sugiura Y, Ikeda K, Nakano M. High membrane curvature enhances binding, conformational changes, and fibrillation of amyloid- $\beta$ on lipid bilayer sur- 
faces. Langmuir. 2015;31(42):11549-11557.

59 . Yuyama K, et al. A potential function for neuronal exosomes: sequestering intracerebral amyloid- $\beta$ peptide. FEBS Lett. 2015;589(1):84-88.

60 . Yuyama K, et al. Decreased amyloid- $\beta$ pathologies by intracerebral loading of glycosphingolipid-enriched exosomes in Alzheimer model mice. J Biol Chem. 2014;289(35):24488-24498.

61. An K, et al. Exosomes neutralize synapticplasticity-disrupting activity of $A \beta$ assemblies in vivo. Mol Brain. 2013;6:47.

62. Dinkins MB, Dasgupta S, Wang G, Zhu G, Bieberich $\mathrm{E}$. Exosome reduction in vivo is associated with lower amyloid plaque load in the 5XFAD mouse model of Alzheimer's disease. Neurobio Aging. 2014;35(8):1792-1800.

63. Grey M, et al. Acceleration of $\alpha$-synuclein aggregation by exosomes. J Biol Chem . 2015;290(5):2969-2982.

64. Asai H, et al. Depletion of microglia and inhibition of exosome synthesis halt tau propagation. Nat Neurosci. 2015;18(11):1584-1593.

65. Joshi P, Benussi L, Furlan R, Ghidoni R, Verderio C. Extracellular vesicles in Alzheimer's disease: friends or foes? Focus on a $\beta$-vesicle interaction. Int J Mol Sci. 2015;16(3):4800-4813.

66. Saman S, et al. Proteins recruited to exosomes by tau overexpression implicate novel cellular mechanisms linking tau secretion with Alzheimer's disease. JAlzheimers Dis. 2014;40(suppl 1):S47-S70.

67. Emmanouilidou E, et al. Cell-produced $\alpha$-synuclein is secreted in a calcium-dependent manner by exosomes and impacts neuronal survival. J Neurosci. 2010;30(20):6838-6851.

68. Danzer KM, et al. Exosomal cell-to-cell transmission of alpha synuclein oligomers. Mol Neurodegener. 2012;7:42.

69. Chutna O, et al. The small GTPase Rab11 colocalizes with $\alpha$-synuclein in intracellular inclusions and modulates its aggregation, secretion and toxicity. Hum Mol Genet. 2014;23(25):6732-6745.

70. Grad LI, Fernando SM, Cashman NR. From molecule to molecule and cell to cell: prion-like mechanisms in amyotrophic lateral sclerosis. Neurobiol Dis. 2015;77:257-265.

71. Basso M, et al. Mutant copper-zinc superoxide dismutase (SOD1) induces protein secretion pathway alterations and exosome release in astrocytes: Implications for disease spreading and motor neuron pathology in amyotrophic lateral sclerosis. J Biol Chem. 2013;288(22):15699-15711.

72. Sephton CF, Yu G. The function of RNAbinding proteins at the synapse: implications for neurodegeneration. Cell Mol Life Sci. 2015;72(19):3621-3635.

73. Villarroya-Beltri C, et al. Sumoylated hnRNPA2B1 controls the sorting of miRNAs into exosomes through binding to specific motifs. Nat Commun. 2013;4:2980.

74. Li W, et al. Human endogenous retrovirus-K contributes to motor neuron disease. Sci Transl Med. 2015;7(307):307ra153.

75. Glass CK, Saijo K, Winner B, Marchetto MC, Gage $\mathrm{FH}$. Mechanisms underlying inflammation in neurodegeneration. Cell. 2010;140(6):918-934.

76. Ridder K, et al. Extracellular vesicle-mediated transfer of genetic information between the hematopoietic system and the brain in response to inflammation. PLoS Biol. 2014;12(6):e1001874.

77. Sáenz-Cuesta M, Osorio-Querejeta I, Otaegui D. Extracellular vesicles in multiple sclerosis: what are they telling us? Front Cell Neurosci. 2014;8:100

78. Yang Y, et al. Cerebrospinal fluid particles in Alzheimer disease and Parkinson disease. J Neuropathol Exp Neurol. 2015;74(7):672-687.

79. Saman S, et al. Exosome-associated tau is secreted in tauopathy models and is selectively phosphorylated in cerebrospinal fluid in early Alzheimer disease. J Biol Chem. 2012;287(6):3842-3849.

80. Verderio C, et al. Myeloid microvesicles are a marker and therapeutic target for neuroinflammation. Ann Neurol. 2012;72(4):610-624.

81. Feneberg E, et al. Limited role of free TDP- 43 as a diagnostic tool in neurodegenerative diseases. Amyotroph Lateral Scler Frontotemporal Degener. 2014;15(5-6):351-356.

82. Ho DH, Yi S, Seo H, Son I, Seol W. Increased DJ-1 in urine exosome of Korean males with Parkinson's disease. Biomed Res Int. 2014;2014:704678.

83. Fraser KB, et al. LRRK2 secretion in exosomes is regulated by 14-3-3. Hum Mol Genet. 2013;22(24):4988-5000.

84. Cocucci E, Racchetti G, Meldolesi J. Shedding microvesicles: artefacts no more. Trends Cell Biol. 2009;19(2):43-51.

85. Shi M, et al. Plasma exosomal $\alpha$-synuclein is likely CNS-derived and increased in Parkinson's disease. Acta Neuropathol. 2014;128(5):639-650.

86. Fiandaca, et al. Identification of preclinical Alzheimer's disease by a profile of pathogenic proteins in neurally derived blood exosomes: a case-control study. Alzheimers Dement. 2015;11(6):600-607.e601.

87. D'Ambrosio A, et al. Peripheral blood biomarkers in multiple sclerosis. Autoimmun Rev. 2015;14(12):1097-1110.

88. Hochberg FH, et al. Glioma diagnostics and biomarkers: an ongoing challenge in the field of medicine and science. Expert Rev Mol Diagn. 2014;14(4):439-452.

89. Nakano I, Garnier D, Minata M, Rak J. Extracellular vesicles in the biology of brain tumour stem cells - implications for inter-cellular communication, therapy and biomarker development. Semin Cell Dev Biol. 2015;40:17-26.

90. Minciacchi VR, et al. Large oncosomes contain distinct protein cargo and represent a separate functional class of tumor-derived extracellular vesicles. Oncotarget. 2015;6(13):11327-11341.

91. Bronisz A, et al. Extracellular vesicles modulate the glioblastoma microenvironment via a tumor suppression signaling network directed by miR-1. Cancer Res. 2014;74(3):738-750.

92. Tian Y, et al. MicroRNA miR-451 downregulates the PI3K/AKT pathway through CAB39 in human glioma. Int JOncol. 2012;40(4):1105-1112.

93. Jaiswal R, et al. Microparticle-associated nucleic acids mediate trait dominance in cancer. FASEBJ. 2012;26(1):420-429.

94. Al-Nedawi K, et al. Intercellular transfer of the oncogenic receptor EGFRvIII by microvesicles derived from tumour cells. Nat Cell Biol. 2008;10(5):619-624.
95. Antonyak MA, et al. Cancer cell-derived microvesicles induce transformation by transferring tissue transglutaminase and fibronectin to recipient cells. Proc Natl Acad Sci U S A. 2011;108(12):4852-4857.

96. Lai CP, et al. Visualization and tracking of tumour extracellular vesicle delivery and RNA translation using multiplexed reporters. Nat Commun. 2015;6:7029.

97. van der Vos KE, et al. Directly visualized glioblastoma-derived extracellular vesicles transfer RNA to microglia/macrophages in the brain. Neuro Oncol. 2016;18(1):58-69.

98. Li CC, et al. Glioma microvesicles carry selectively packaged coding and noncoding RNAs which alter gene expression in recipient cells. RNA Biol. 2013;10(8):1333-1344.

99. Skog J, et al. Glioblastoma microvesicles transport RNA and protein that promote tumor growth and provide diagnostic biomarkers. Nat Cell Biol. 2008;10(12):1470-1476.

100.Stiles CD, Rowitch DH. Glioma stem cells: a midterm exam. Neuron. 2008;58(6):832-846.

101.Suvà $\mathrm{ML}$, et al. Reconstructing and reprogramming the tumor-propagating potential of glioblastoma stem-like cells. Cell. 2014;157(3):580-594.

102. Verhaak RG, et al. Integrated genomic analysis identifies clinically relevant subtypes of glioblastoma characterized by abnormalities in PDGFRA, IDH1, EGFR, and NF1. Cancer Cell. 2010;17(1):98-110.

103. Carone $\mathrm{C}$, et al. In vitro effects of cocaine on tunneling nanotube formation and extracellular vesicle release in glioblastoma cell cultures. $J \mathrm{Mol}$ Neurosci. 2015;55(1):42-50.

104.Sullivan JP, et al. Brain tumor cells in circulation are enriched for mesenchymal gene expression. Cancer Discov. 2014;4(11):1299-1309.

105. Wurdinger T, Deumelandt K, van der Vliet HJ, Wesseling P, de Gruijl TD. Mechanisms of intimate and long-distance cross-talk between glioma and myeloid cells: How to break a vicious cycle. Biochim Biophys Acta. 2014;1846(2):560-575.

106. Harshyne LA, Nasca BJ, Kenyon LC, Andrews DW, Hooper DC. Serum exosomes and cytokines promote a T-helper cell type 2 environment in the peripheral blood of glioblastoma patients [published online ahead of print July 14, 2015]. Neuro Oncol. doi: 10.1093/neuonc/nov107.

107. de Vrij J, et al. Glioblastoma-derived extracellular vesicles modify the phenotype of monocytic cells. Int JCancer. 2015;137(7):1630-1642.

108. Perry JR. Thromboembolic disease in patients with high-grade glioma. Neuro Oncol. 2012;14(suppl 4):iv73-iv80.

109. Sartori MT, et al. Prothrombotic state in glioblastoma multiforme: an evaluation of the procoagulant activity of circulating microparticles. J Neurooncol. 2011;104(1):225-231.

110.D’Asti E, Magnus N, Meehan B, Garnier D, Rak J. Genetic basis of thrombosis in cancer. Semin Thromb Hemost. 2014;40(3):284-295.

111. Noerholm M, et al. RNA expression patterns in serum microvesicles from patients with glioblastoma multiforme and controls. BMC Cancer. 2012;12:22.

112. Muller L, et al. Exosomes isolated from plasma of glioma patients enrolled in a vaccination 
trial reflect antitumor immune activity and might predict survival. Oncoimmunology. 2015;4(6):e1008347.

113. Manterola L, et al. A small noncoding RNA signature found in exosomes of GBM patient serum as a diagnostic tool. Neuro Oncol. 2014;16(4):520-527.

114. Chen WW, et al. BEAMing and droplet digital PCR analysis of mutant IDH1 mRNA in glioma patient serum and cerebrospinal fluid extracellular vesicles. Mol Ther Nucleic Acids. 2013;2:e109.

115. Akers JC, et al. MiR-21 in the extracellular vesicles (EVs) of cerebrospinal fluid (CSF): a platform for glioblastoma biomarker development. PLoS One. 2013;8(10):e78115.

116. Shao $\mathrm{H}$, et al. Protein typing of circulating microvesicles allows real-time monitoring of glioblastoma therapy. Nat Med. 2012;18(12):1835-1840.

117. Shao H, et al. Chip-based analysis of drug resistance of glioblastoma using exosomes. Nat Commun. 2015;6:6999.

118. Wei X, et al. Mesenchymal stem cells: a new trend for cell therapy. Acta Pharmacol Sin. 2013;34(6):747-754.

119. Kim JM, et al. Systemic transplantation of human adipose stem cells attenuated cerebral inflammation and degeneration in a hemorrhagic stroke model. Brain Res. 2007;1183:43-50.

120. Li Y, et al. Human marrow stromal cell therapy for stroke in rat: neurotrophins and functional recovery. Neurology. 2002;59(4):514-523.

121. Inoue $Y$, et al. Subretinal transplantation of bone marrow mesenchymal stem cells delays retinal degeneration in the RCS rat model of retinal degeneration. Exp Eye Res. 2007;85(2):234-241.

122.Rani S, Ryan AE, Griffin MD, Ritter T. Mesenchymal stem cell-derived extracellular vesicles: toward cell-free therapeutic applications. $\mathrm{Mol}$ Ther. 2015;23(5):812-823.

123. Zhang B, et al. Mesenchymal stem cells secrete immunologically active exosomes. Stem Cells Dev. 2014;23(11):1233-1244.

124. Xin H, et al. MiR-133b promotes neural plasticity and functional recovery after treatment of stroke with multipotent mesenchymal stromal cells in rats via transfer of exosome-enriched extracellular particles. Stem Cells. 2013;31(12):2737-2746.

125. Xin H, et al. Systemic administration of exosomes released from mesenchymal stromal cells promote functional recovery and neurovascular plasticity after stroke in rats. J Cereb Blood Flow Metab. 2013;33(11):1711171-1711175.

126. Montecalvo A, et al. Mechanism of transfer of functional microRNAs between mouse dendritic cells via exosomes. Blood. 2012;119(3):756-766.

127. Pusic AD, Pusic KM, Clayton BL, Kraig RP. IFN $\gamma$ stimulated dendritic cell exosomes as a potential therapeutic for remyelination. J Neuroimmunol. 2014;266(102):12-23.

128. György B, Fitzpatrick Z, Crommentuijn MH, $\mathrm{Mu}$ D, Maguire CA. Naturally enveloped AAV vectors for shielding neutralizing antibodies and robust gene delivery in vivo. Biomaterials. 2014;35(26):598-609.

129. Tian Y, et al. A doxorubicin delivery platform using engineered natural membrane vesicle exosomes for targeted tumor therapy. Biomaterials. 2014;35(7):2383-2390.

130. Alvarez-Erviti L, et al. Delivery of siRNA to the mouse brain by systemic injection of targeted exosomes. Nat Biotechnol. 2011;29(4):341-345.

131. Ohno S, et al. Systemically injected exosomes targeted to EGFR deliver antitumor microRNA to breast cancer cells. Mol Ther. 2013;21(1):185-191.

132. Mizrak A, et al. Genetically engineered microvesicles carrying suicide mRNA/protein inhibit schwannoma tumor growth. Mol Ther. 2013;21(1):101-108.

133. Kanada M, et al. Differential fates of biomolecules delivered to target cells via extracellular vesicles. Proc Natl Acad Sci U S A. 2015;112(12):E1433-E1442.

134. Mangeot PE, et al. Protein transfer into human cells by VSV-G-induced nanovesicles. Mol Ther. 2011;19(9):1656-1666.

135. Sun D, et al. A novel nanoparticle drug delivery system: the anti-inflammatory activity of curcumin is enhanced when encapsulated in exosomes. Mol Ther. 2010;18(9):1606-1614.

136. Maguire CA, et al. Microvesicle-associated AAV vector as a novel gene delivery system. Mol Ther. 2012;20(5):960-971.

137. Zhuang X, et al. Treatment of brain inflammatory diseases by delivering exosome encapsulated anti-inflammatory drugs from the nasal region to the brain. Mol Ther. 2011;19(10):1769-1779.

138. Munoz JL, et al. Delivery of functional anti-miR-9 by mesenchymal stem cell-derived exosomes to glioblastoma multiforme cells conferred chemosensitivity. Mol Ther Nucleic Acids. 2013;2:e126.

139. Yang $\mathrm{T}$, et al. Exosome delivered anticancer drugs across the blood-brain barrier for brain cancer therapy in Danio rerio. Pharm Res. 2015;32(6):2003-2014.

140. Maguire AM, et al. Safety and efficacy of gene transfer for Leber's congenital amaurosis. $N$ Engl JMed. 2008;358(21):2240-2248.

141. Maguire C, Ramirex SH, Merkel SF, Sena-Esteves $\mathrm{M}$, Breakefield XO. Gene therapy for the nervous system: challenges and new strategies. Neurotherapeutics. 2014;11(4):817-839.

142. Mingozzi F, High KA. Therapeutic in vivo gene transfer for genetic disease using AAV: progress and challenges. Nat Rev Genet. 2011;12(1):341-355.

143. Scallan CD, et al. Human immunoglobulin inhibits liver transduction by AAV vectors at low AAV2 neutralizing titers in SCID mice. Blood. 2006;107(5):1810-1817.

144.Feng Z, et al. A pathogenic picornavirus acquires an envelope by hijacking cellular membranes. Nature. 2013;496(7445):367-371.

145. Ramakrishnaiah V, et al. Exosome-mediated transmission of hepatitis $\mathrm{C}$ virus between human hepatoma Huh7.5 cells. Proc Natl Acad Sci U S A. 2013;110(32):13109-13113.

146.Weinberg MS, Samulski RJ, McCown TJ. Adenoassociated virus (AAV) gene therapy for neurological disease. Neuropharmacology. 2013;69:82-88.

147. Lai CP, Breakefield XO. Role of exosomes/ microvesicles in the nervous system and use in emerging therapies. Front Physiol. 2012;3:228.

148. Yuyama K, Sun H, Mitsutake S, Igarashi Y. Sphingolipid-modulated exosome secretion promotes the clearance of amyloid- $\beta$ by microglia. J Biol Chem. 2012;287(14):10977-10989. 